\title{
Biomasa, producción de semillas y fenología de Chusquea montana tras su floración masiva y sincrónica en el Parque Nacional Puyehue, Chile
}

\author{
Biomass, seed production and phenology of Chusquea montana \\ after a massive and synchronous flowering event in Puyehue National Park, Chile
}

\author{
Alejandro de la Fuente ${ }^{\text {a*, }}$, Nicolás Pacheco ${ }^{\text {a }}$ \\ *Autor de correspondencia: a Departamento de Recursos Naturales, \\ Parque Nacional Puyehue, Osorno, Chile, alejandrofuentepinero@gmail.com
}

\begin{abstract}
SUMMARY
In spring of 2015, a massive and synchronous flowering and seeding event of Chusquea montana began in Puyehue National Park, Lake District. We assessed above ground biomass and temporal pattern of productivity and viability of seeds by sampling eight plots and 20 collection boxes, respectively. In addition, we recorded the different phenological stages of the species during this event. The study area was in the sector of Antillanca, dominated by a mixed evergreen-deciduous Nothofagus forest. Biomass values were $33.50 \mathrm{Mg} \mathrm{ha}^{-1}$ fresh weight and $17.66 \mathrm{Mg} \mathrm{ha}^{-1}$ dry weight. The peak values of seed production and viability were $146.86 \times 10^{6} \pm 130.54 \times 10^{6}$ seeds $\mathrm{ha}^{-1}$ and $87.5 \%$, respectively. The phenological features observed were similar to those observed for Chusquea quila; nevertheless, mountain climate, where Chusquea montana occurs, was reflected by some differences in maturation and seeding times.
\end{abstract}

Key words: seeds, biomass, phenology, Chusquea montana.

\section{RESUMEN}

En primavera de 2015 se inició el evento de floración masiva y sincrónica de Chusquea montana en el Parque Nacional Puyehue, región de Los Lagos. Se estimó la biomasa aérea y se evaluó el patrón temporal de producción y viabilidad de semillas mediante el muestreo de ocho parcelas y 20 cajones recolectores, respectivamente. Además, se registraron las diferentes etapas fenológicas de la especie durante su evento reproductivo. El estudio se realizó en el sector de Antillanca, dominado por bosques mixtos siempreverdecaducifolio de Nothofagus spp. La biomasa alcanzó 33,50 $\mathrm{Mg} \mathrm{ha}^{-1}$ en peso fresco y 17,66 $\mathrm{Mg}^{-1}$ en peso seco. La producción y viabilidad de semillas alcanzó su máximo con 146,86 × $10^{6} \pm 130,54 \times 10^{6}$ semillas ha-1 y $87,5 \%$, respectivamente. Las características fenológicas siguieron esencialmente las descritas previamente para Chusquea quila, sin embargo, el clima de montaña en el que Chusquea montana se desarrolla determinó diferencias en los tiempos de maduración y diseminación de semillas.

Palabras clave: semillas, biomasa, fenología, Chusquea montana.

\section{INTRODUCCIÓN}

El género Chusquea Kunth (Poaceae: Bambusoideae), distribuido desde México hasta Chile y Argentina, es uno de los géneros más diversos y numerosos en especies dentro de las bambúceas (McClure 1993, Clark 1997). En Chile se han descrito 12 especies, distribuidas desde la región

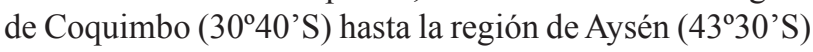
(Parodi 1945).

Una de las características que define a muchas de las especies de este género radica en un ciclo vital supranual, probablemente regulado por factores endogenéticos (Janzen 1976), que puede oscilar entre 15 y 70 años (Matthei 1998, González y Donoso 1999, Guerreiro 2014). Después de un largo periodo de estado vegetativo, este singular ciclo de vida termina con un único evento reproductivo consistente en una floración, semillación, muerte masiva y sincrónica (McClure 1993).

Asociado a estos eventos, se acumulan grandes cantidades de biomasa seca que constituye una fuente de combustible que puede aumentar la probabilidad de incendio (p.e., "Cerro Chamuscado", lago Rupanco, en 1940, Pacheco 1993). Además, a la producción masiva de semillas normalmente le siguen explosiones demográficas de granívoros, conocidas como "ratadas" (Janzen 1976, Murúa et al. 1996).

En primavera de 2015 se inició el florecimiento de Chusquea montana Phil. f. montana (denominada anteriormente Chusquea tenuiflora, Matthei 1998) en el Parque Nacional Puyehue, Región de Los Lagos. Chusquea montana es una especie dominante del sotobosque de los bosques mixtos siempreverde-caducifolios cordilleranos, 
desarrollándose entre los 750-1.200 m s.n.m. (Veblen et al. 1977, Matthei 1998, Holz y Veblen 2006, Pacheco 2013). La floración afectó a toda la población de C. montana presente en el Parque a excepción del parche ubicado en las inmediaciones del complejo volcánico Puyehue - Cordón Caulle, cuyo sotobosque fue arrasado por la caída de ceniza durante la erupción de 2011 (Swanson et al. 2016). Otro registro de floración de esta especie fue reportado en 2001 por Holz y Veblen (2006), en el paso Carirriñe, Región de Los Ríos, asociada a bosques de lenga (Nothofagus pumilio (Poepp. et Endl.) Krasser 1896).

Aunque las floraciones de bambúceas han sido reportadas durante siglos en otras partes del mundo, en América los reportes han sido escasos. Algunos investigadores han estudiado los eventos reproductivos de especies del género Chusquea en Chile y Argentina, describiendo la fenología, cuantificando la biomasa y/o evaluando la productividad de semillas (Chusquea culeou E. Desv., Veblen et al. 1979, Sage et al. 2007, Marchesini et al. 2009; Chusquea quila Kunth, González y Donoso 1999, González 2001), pero no hay antecedentes sobre $C$. montana durante su floración.

Considerando la importancia de este evento, el desconocimiento y falta de información de los ciclos reproductivos de C. montana, este estudio tiene como objetivos (1) cuantificar la biomasa aérea y la productividad de semillas de la especie durante su evento reproductivo en el Parque Nacional Puyehue, y (2) describir las etapas fenológicas de la misma durante su floración masiva y sincrónica.

\section{MÉTODOS}

Área de estudio. El área de estudio se ubica en el sector de Antillanca $\left(40^{\circ} 78^{\prime} \mathrm{S}\right.$ y $72^{\circ} 22^{\prime} \mathrm{O}$, figura 1), entre los 850 $1.000 \mathrm{~m}$ s.n.m., abarcando el bosque mixto siempreverdecaducifolio, compuesto por lenga ( $N$. pumilio) y coihue de Magallanes (Nothofagus betuloides (Mirb.) Oerst. 1871); y la parte alta del bosque siempreverde, compuesto por coihue (Nothofagus dombeyi (Mirb.) Oerst. 1871) y mañíos (Saxegothaea conspicua Lindl. 1851, Podocarpus nubigenus Lindl. 1851). En el estrato arbustivo acompañante de C. montana se puede encontrar chaura (Gaultheria phillyreifolia (Pers.) Sleumer 1936), canelo enano (Drimys andina (Reiche) RA Rodr. et Quez. 1991), caña-colihue (C. culeou) y taique (Desfontainia spinosa Ruiz et Pav. 1799).

El sector presenta un clima de montaña, con una temperatura promedio anual de $4,1^{\circ} \mathrm{C}$, con una mínima de $-2,5^{\circ} \mathrm{C}$ en julio y una máxima de $13,9^{\circ} \mathrm{C}$ en febrero. La precipitación anual es alta, alcanzando los $5.913 \mathrm{~mm}$, con un mínimo de $286,4 \mathrm{~mm}$ en diciembre y un máximo de $942,8 \mathrm{~mm}$ en junio (1997-2001, estación meteorológica temporal de Antillanca).

Cuantificación de biomasa aérea. Se determinó por conteo directo el número de culmos en ocho parcelas de $25 \mathrm{~m}^{2}$ en octubre de 2016, cuando la planta se encontraba verde y ac-

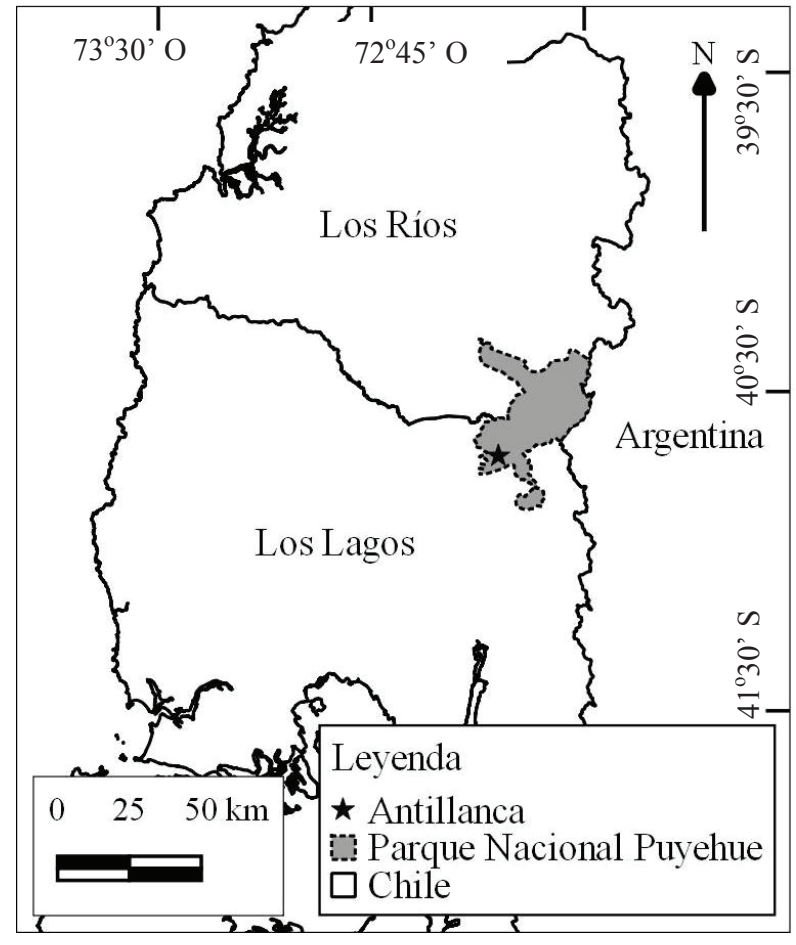

Figura 1. Localización del Parque Nacional Puyehue y área de estudio en Antillanca.

Location of Puyehue National Park and the study area in the sector of Antillanca.

tiva. Las parcelas se ubicaron paralelas al camino que cruza el valle de Antillanca, dejando $2 \mathrm{~m}$ de espacio desde la orilla del camino. La primera parcela se estableció a $890 \mathrm{~m}$ s.n.m., donde C. montana empieza a dominar absolutamente el sotobosque. Las siguientes parcelas se establecieron de forma ascendente separadas entre 50-100 m. Se extrajo, mediante corte a ras de suelo, la parte aérea de cinco culmos al azar por parcela y fueron pesados en terreno para obtener el peso fresco. El peso seco se obtuvo a partir del peso promedio del culmo fresco y la relación de peso seco/peso fresco, calculada a partir del promedio de tres muestras de un kilogramo de culmos frescos secados en dos tiempos, durante un total de seis semanas, primero al sol y posteriormente mediante estufa casera sin temperatura controlada. Con las medidas de peso fresco y seco de un culmo y la estimación del número de culmos por hectárea obtenida mediante un factor simple de expansión, se pudo determinar la biomasa aérea fresca y seca que presentaba la especie por hectárea (media \pm desviación estándar).

Producción y viabilidad de semillas. Se emplazaron al azar 20 cajones recolectores de $0,25 \mathrm{~m} \mathrm{x} \mathrm{0,50} \mathrm{m}$ x $0,20 \mathrm{~m}$ de alto en el área de estudio en diciembre de 2016, cuando la dispersión de semillas aún no había comenzado; cumpliendo la premisa de que la superficie de cajón estuviese 
cubierta completamente por culmos floridos. Tenían instalados una malla de menos de $2 \mathrm{~mm}$ de poro para la recolección de semillas y que, a su vez, dejara libre paso al agua; y una malla en la parte superior de $50 \mathrm{~mm}$ de espacio para reducir la depredación por aves. El número de cajones siguió los supuestos de Newbold (1967) para el muestreo homogéneo y la reducción de la varianza. La recolección de semillas se efectuó semanalmente durante un lapso de 12 semanas (entre enero y abril de 2017); donde se asumió que valores cercanos al $0 \%$ de viabilidad suponía el fin de la semillación, terminando el monitoreo. Después de cada colecta se contaron las semillas y se determinó la viabilidad aplicando una prueba de corte a 10 semillas al azar por cajón recolector, considerándose no viables aquellas huecas o sin endosperma (Hartmann y Kester 1975).

Diagrama fenológico. Siguiendo la línea establecida por González (2001), se registró mensualmente, durante un periodo de 19 meses (entre octubre de 2015 y abril de 2017), las diferentes etapas fenológicas de C. montana durante su evento reproductivo (follaje verde, floración, formación y maduración de semillas, senescencia y muerte de la planta, diseminación de semillas, derrumbe y descomposición) en las parcelas delimitadas previamente.

\section{RESULTADOS}

Cuantificación de biomasa aérea. El conteo directo de culmos promedió $115.500 \pm 25.647,1$ culmos por hectárea, variando entre 157.600 y 82.000 culmos ha-1. El peso promedio de culmos frescos y secos por hectárea (calculado por la relación: peso seco/peso fresco, con resultado medio de 0,527 ), determinó un promedio de biomasa fresca y seca de $33,50 \pm 7,12$ y $17,66 \pm 3,75 \mathrm{Mg} \mathrm{ha}^{-1}$, respectivamente.

Producción y viabilidad de semillas. Durante las 12 semanas de dispersión de semillas, $C$. montana presentó una marcada oscilación en la producción, alcanzando su máximo valor la séptima semana, con 146,86 x 10 $\pm 130,54$ x $10^{6}$ semillas por hectárea (cuadro 1). La viabilidad presentó una dinámica más uniforme, siendo el periodo de máxima viabilidad durante las semanas centrales de verano, alcanzando su máximo valor la cuarta semana, con un 87,5\% (cuadro 1); y reduciéndose a finales de febrero (octava semana), cuando la mayor parte de las semillas viables ya han caído y la diseminación estaba terminando. Considerado todo el periodo analizado, el número de semillas viables por hectárea fue de 253,88 x 106 (cuadro 1).

Fenología de Chusquea montana. En primavera de 2015 comenzó la floración gregaria de $C$. montana. Durante este proceso, hasta la fase final de maduración de semillas (primavera de 2016), la especie se encontraba verde y activa, pero sin formar nuevos culmos o follaje. A partir de entonces, comenzó la fase de senescencia que acabó con la muerte de la planta durante primavera-verano de 2017. El proceso de maduración de semillas se extendió más de un año, desde finales de primavera de 2016 hasta el verano de 2017, donde empezó la dispersión de semillas, prolongándose durante 12 semanas, hasta principios de otoño de 2017, momento en el que los individuos comenzaron a desmoronarse y descomponerse (figura 2 y anexo).

Cuadro 1. Producción y viabilidad semanal de semillas de Chusquea montana durante la diseminación.

Weekly production and viability of seeds of Chusquea montana during the dispersal event.

\begin{tabular}{ccccc}
\hline Fecha & $\mathrm{N}^{\mathrm{o}}$ semana & Semillas ha $^{-1} \pm$ D.E. $\left(\mathrm{x} 10^{6}\right)$ & Viabilidad $(\%)^{\circ}$ & Semillas viables ha $^{-1}\left(\mathrm{x} 10^{6}\right)$ \\
\hline $10 / 01 / 2017$ & 1 & $24,86 \pm 16,01$ & 68,0 & 16,9 \\
$17 / 01 / 2017$ & 2 & $9,88 \pm 4,43$ & 63,0 & 6,1 \\
$24 / 01 / 2017$ & 3 & $19,13 \pm 12,13$ & 69,5 & 20,61 \\
$31 / 01 / 2017$ & 4 & $23,55 \pm 19,08$ & 87,5 & 62,38 \\
$07 / 02 / 2017$ & 5 & $77,01 \pm 66,96$ & 81,0 & 6,94 \\
$14 / 02 / 2017$ & 6 & $9,19 \pm 6,70$ & 75,5 & 102,8 \\
$21 / 02 / 2017$ & 7 & $146,86 \pm 130,54$ & 70,0 & 11,11 \\
$28 / 02 / 2017$ & 8 & $24,69 \pm 19,62$ & 42,5 & 10,04 \\
$07 / 03 / 2017$ & 9 & $41,82 \pm 32,59$ & 24,0 & 3,44 \\
$14 / 03 / 2017$ & 10 & $43,02 \pm 18,98$ & 8,0 & 0,21 \\
$21 / 03 / 2017$ & 11 & $6,95 \pm 4,81$ & 3,0 & 0,06 \\
$28 / 03 / 2017$ & 12 & $4,18 \pm 2,92$ & 1,5 & 253,88 \\
\hline
\end{tabular}




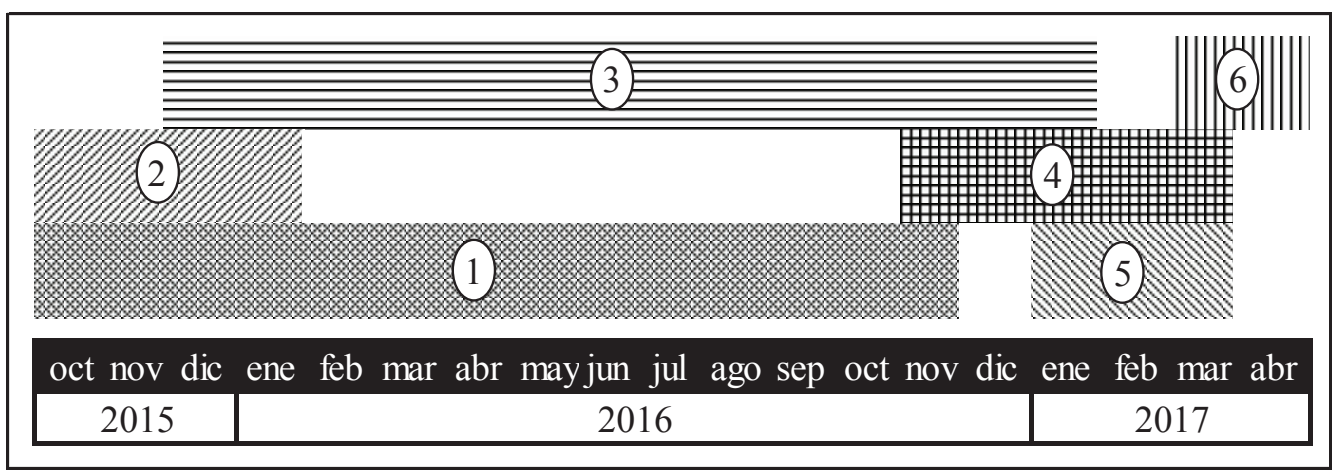

Figura 2. Etapas fenológicas de Chusquea montana durante el evento reproductivo en el Parque Nacional Puyehue (años 2015-2017): 1) follaje verde, 2) floración, 3) formación y maduración de semillas, 4) senescencia y muerte de la planta, 5) diseminación de semillas, 6) derrumbe y descomposición.

Phenological stages of Chusquea montana during a reproductive event in Puyehue National Park (years 2015-2017): 1) green foliage, 2) flowering, 3) growth and maturing of seeds, 4) senescence and death of plants, 5) seeding, 6) collapse and decomposition.

\section{DISCUSIÓN}

Presentando una gran cantidad de culmos por hectárea, Chusquea montana mostró en Antillanca valores cercanos a los estimados en Remeco y Choshuenco (región de Los Ríos), sectores donde la especie abarca $>80 \%$ del sotobosque (Veblen 1982). Además, presentó valores similares a especies afines, como C. culeou, estimados para bosques transandinos (Sage et al. 2007). Por otra parte, Veblen et al. (1979) estiman en Antillanca más del doble de culmos por hectárea de C. montana, pero debido a que el estudio se realiza fuera del evento reproductivo, incluye culmos menos desarrollados y nuevos $(<1$ año), presentando valores de biomasa inferiores comparado con este estudio. En cambio, como consecuencia del gatillazo del evento reproductivo que inhibe la formación de nuevos culmos, la población evaluada en este estudio se constituía fundamentalmente por culmos completamente desarrollados $(2,65 \mathrm{~m}$ en promedio, datos no publicados). Por lo tanto, las diferencias encontradas para la misma población en los diferentes estudios estarían ligadas al estado de desarrollo poblacional, siendo una población madura aquella que concentra gran cantidad de biomasa en culmos grandes, asegurando una mayor producción de semillas (Janzen 1976). Sin embargo, la biomasa aérea seca asociada a la gran cobertura que C. montana presenta en Antillanca, fue baja comparada con lo estimado para C. culeou (Veblen et al. 1979, Marchesini et al. 2009), debido a la diferencia de tamaño entre ambas especies, siendo C. culeou 10 veces más pesada (Veblen et al. 1979, Matthei 1998, Pacheco 2013).

La maduración de semillas está regulada en gran parte por factores externos, siendo la temperatura un factor limitante. Temperaturas templadas y cálidas, entre $6-30^{\circ} \mathrm{C}$, estimulan la maduración, pero temperaturas fuera de ese rango suelen inhibir el proceso. Una de las características que diferencia a C. montana de otras especies del género es el clima de montaña en el que se desarrolla, presentando veranos más cortos y temperaturas medias anuales de hasta $7{ }^{\circ} \mathrm{C}$ más bajas que en climas a cotas inferiores. Esto se traduce en periodos de maduración de semillas más largos y procesos de diseminación más tardíos y concentrados en el tiempo en comparación con C. quila, que se desarrolla en un clima templado (González y Donoso 1999, González 2001). Siguiendo los factores que intervienen en los procesos de diseminación de semillas, en ambas especies se concentran en los meses centrales del verano, donde las semillas alcanzan su máxima madurez siendo diseminadas de forma natural y masiva (González y Donoso 1999).

Las etapas fenológicas de $C$. montana siguen esencialmente las descritas para C. quila (González 2001). Sin embargo, hay ciertas diferencias en los tiempos de desarrollo de dichas etapas (aumento en 13 semanas del tiempo de maduración, retraso y reducción del periodo de dispersión en 12 y 68 semanas, respectivamente), principalmente debido a las características climáticas dispares mencionadas anteriormente.

La producción total de semillas viables por hectárea resultante del evento reproductivo de especies del género Chusquea, oscila dentro de un amplio rango que varía desde $195,5 \times 10^{6}$ semillas por hectárea para C. quila (González y Donoso 1999) hasta $810 \times 10^{6}$ semillas por hectárea para C. culeou (Sage et al. 2007), pasando por $254 \times 10^{6}$ semillas por hectárea para C. montana (este estudio). Esto podría revelar una mayor productividad en especies del género Chusquea que presentan una morfología tipo "vara recta", con ramificaciones erectas paralelas al culmo, como C. culeou y C. montana; siendo menor en las especies trepadoras facultativas, que presentan un gran número de ramificaciones dobladas en su base en $90-180^{\circ}$ con respecto al culmo, como C. quila (Matthei 1998). La diferencia entre C. culeou y C. montana podría atribuirse a las inherentes al tamaño comentadas anteriormente. 


\section{AGRADECIMIENTOS}

Agradecemos a guardaparques del PNP por su inestimable apoyo, a Mauro González y Jaime Rau por la revisión del manuscrito.

\section{REFERENCIAS}

Clark LG. 1997. Diversity, biogeography and evolution of Chusquea. In Chapman GP ed. The bamboos. Linnean Society Symposium Series $N^{\circ} 19$. Londres, Inglaterra. Academic Press. p. 33-44.

González M, C Donoso. 1999. Producción de semillas y hojarasca en Chusquea quila (Poaceae: Bambusoideae), posterior a su floración sincrónica en la zona centro-sur de Chile. Revista Chilena Historia Natural 72(2): 169-180.

González M. 2001. Fenología de Chusquea quila durante su floración gregaria en la zona centro-sur de Chile. Bosque 22(2): 45-51.

Guerreiro C. 2014. Flowering cycles of woody bamboos native to southern South America. Journal of plant research 127(2): 307-313. DOI:10.1007/s10265-013-0593-Z

Hartmann HT, DE Kester. 1975. Plant propagation. Principles and practices. Third edition. New Jersey, USA. Prentice Hall. 662 p.

Holz CA, T Veblen. 2006. Tree regeneration responses to Chusquea montana bamboo die-off in a subalpine Nothofagus forest in the southern Andes. Journal of Vegetation Science 17(1): 19-28.

DOI: $10.1111 / \mathrm{j} .1654-1103.2006 . t b 02419 . x$

Janzen DH. 1976. Why bamboos wait so long to flower. Annual Review of Ecology and Systematics 7(1): 347-391.

Marchesini V, O Sala, A Austin. 2009. Ecological consequences of a massive flowering event of bamboo (Chusquea culeou) in a temperate forest of Patagonia, Argentina. Journal of Vegetation Science 20(3):424-432. DOI: 10.1111/j.16541103.2009.05768.x

Matthei O. 1998. Las especies del genero Chusquea Kunth (Poaceae: Bambusoideae), que crecen en la X Región, Chile.
Gayana Botánica 54(2): 199-220.

McClure FA. 1993. The Bamboos. Washington DC, USA. Smithsonian Institution Press. 345 p.

Murúa R, LA González, M González, C Jofre. 1996. Efectos del florecimiento del arbusto Chusquea quila Kunth (Poaceae) sobre la demografía de poblaciones de roedores de los bosques templados fríos del sur chileno. Boletín de la Sociedad de Biología 67: 37-42.

Newbold PJ. 1967. Methods for estimating the primary production of forests. IBP Handbook $N^{\circ} 2$. Oxford and Edinburgh, UK. Blackwell Scientific Publications. 62 p.

Pacheco N. 1993. Floración de las Quilas. Boletín Flora, Fauna y Aves Silvestres. Santiago, Chile. FAO-PNUMA. p. 34-35.

Pacheco N. 2013. Chusquea (Poaceae) en el Parque Nacional Puyehue, Región de Los Lagos, Chile. Chloris Chilensis 16(1).

Parodi RL. 1945. Sinopsis de las gramíneas chilenas del género Chusquea. Revista Universitaria 30: 61-71.

Sage RD, OP Pearson, J Sanguinetti, AK Pearson. 2007. Ratada 2001: A rodent outbreak following the flowering of bamboo (Chusquea culeou) in southwestern Argentina. In Kelt DA, EP Lessa, J Salazar-Bravo, JL Patton eds. The quintessential naturalist: Honoring the life and legacy of Oliver P. Pearson. Berkeley, California. University of California Press. p. 134-177.

Swanson FJ, J Jones, C Crisafulli, ME González, A Lara. 2016. Puyehue-Cordón Caulle eruption of 2011: tephra fall and initial forest responses in the Chilean Andes. Bosque 37(1): 85-96. DOI: $10.4067 /$ S0717-92002016000100009

Veblen TT, DH Ashton, FM Schlegel, AT Veblen. 1977. Distribution and dominance of species in the understory of a mixed evergreen-deciduous Nothofagus forest in south-central Chile. The Journal of Ecology 65: 815-830.

Veblen TT, F Schlegel, B Escobar. 1979. Biomasa y producción primaria de Chusquea culeou Desv. y Chusquea tenuiflora Phi 1. en el sur de Chile. Bosque 3(1): 47-56.

Veblen TT. 1982. Growth patterns of Chusquea bamboos in the understory of Chilean Nothofagus forest and their influences in forest dynamics. Bulletin of the Torrey Botanical Club 109: 474-487. 
ANEXO
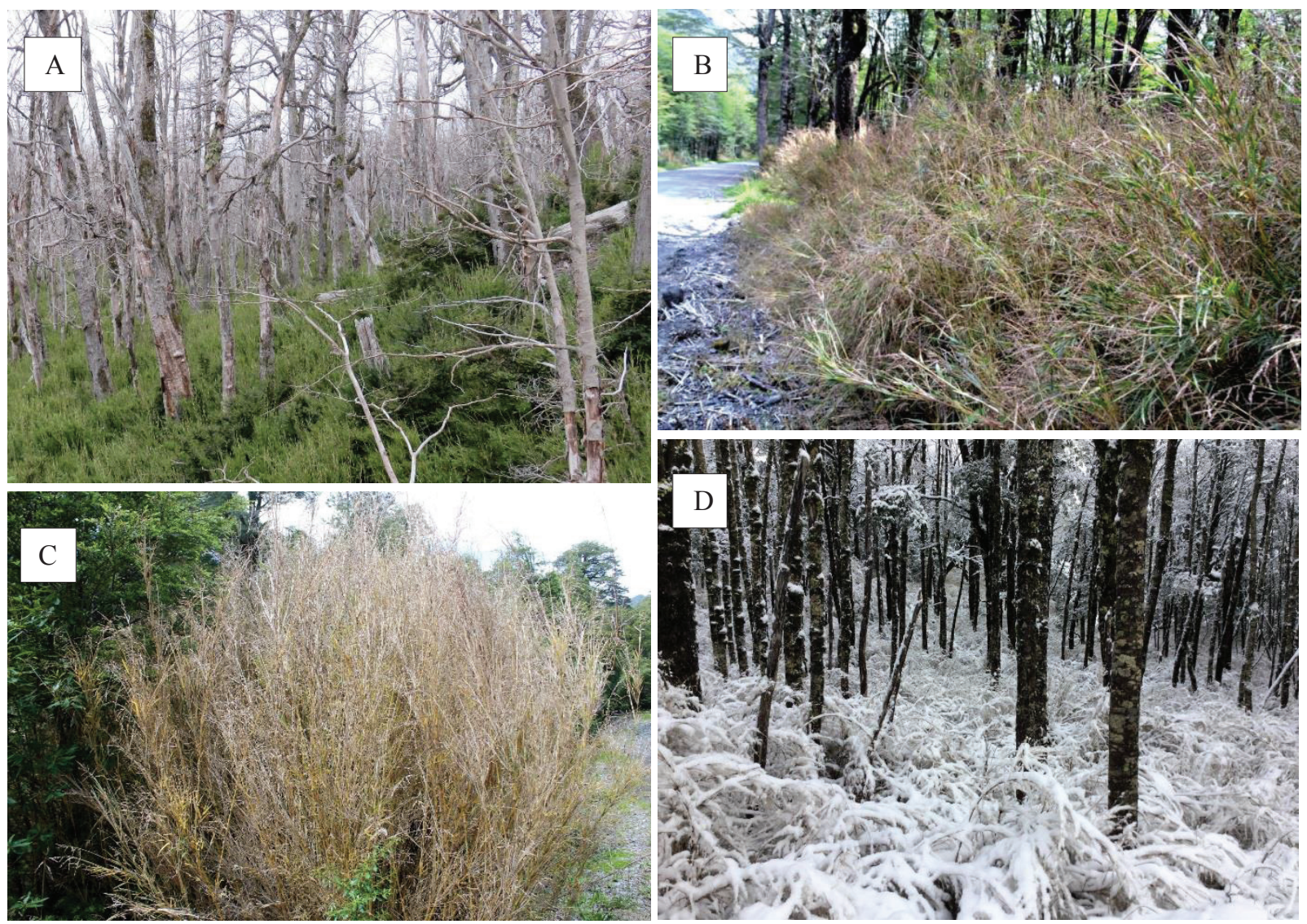

A) Estado vegetativo de Chusquea montana f. montana.

B) Estado florido de Chusquea montana f. montana.

C) Estado senescente. Culmos con semillas de Chusquea montana f. montana.

D) Derrumbe y descomposición de Chusquea montana f. montana. 